Mots. Les langages du politique

$100 \mid 2012$

Chiffres et nombres dans l'argumentation politique

\title{
Le langage des chiffres en politique
}

Paul Bacot, Dominique DESMARCHELIER et Sylvianne Rémi-Giraud

\section{(2) OpenEdition}

Journals

Édition électronique

URL : https://journals.openedition.org/mots/20977

DOI : $10.4000 /$ mots. 20977

ISSN : 1960-6001

Éditeur

ENS Éditions

Édition imprimée

Date de publication : 15 décembre 2012

Pagination : 5-14

ISBN : 978-2-84788-387-9

ISSN : 0243-6450

Référence électronique

Paul Bacot, Dominique DESMARCHELIER et Sylvianne Rémi-Giraud, "Le langage des chiffres en politique », Mots. Les langages du politique [En ligne], 100 | 2012, mis en ligne le 15 décembre 2014 consulté le 22 avril 2022. URL : http://journals.openedition.org/mots/20977 ; DOI : https://doi.org/ $10.4000 /$ mots. 20977

(c) ENS Éditions 


\section{Le langage des chiffres en politique}

Il n'est bien sûr nul besoin de rappeler que dans statistique, il y a État. On sait le faible apport de l'étymologie à l'étiologie. Il faut donc se demander pourquoi les nombres sont omniprésents dans le discours politique - et donc bien audelà du discours étatique. Sur ce point, la littérature est abondante, marquée notamment par l'œuvre d'Alain Desrosières (1993, 2008).

Il y a un siècle déjà, Max Webervoyait dans une rationalisation croissante de l'action publique une caractéristique majeure des États modernes. La bureaucratie qui incarne ce mouvement historique tire sa légitimité de sa compétence - un savoir et un savoir-faire, une science et une technique, qui se nourrissent de chiffres. Ceux-ci vont devenir un marqueur déterminant du langage qu'elle utilise. Le tournant dit «néolibéral» de ces dernières décennies, tout entier fondé sur l'hégémonie supposée de la rationalité individuelle, va placer l'évaluation des performances au cœur de l'action publique. Plus largement, l'impression domine de nos jours que les chiffres ont colonisé le politique sous toutes ses formes. Si l'on «gouverne par les instruments " (Lascoumes, Le Galès, 2004), et notamment par l'instrument statistique, ce sont tous les acteurs politiques qui manient les données quantifiées dans tous les compartiments de leur jeu.

Comme un clin d'œil à cette tendance lourde de l'évolution du discours politique, ce numéro spécial, le centième de la revue, est consacré aux chiffres et aux nombres en politique. Cette thématique a suscité une forte adhésion de la part de chercheurs venant d'horizons disciplinaires différents. L'éclairage vient à la fois des sciences du langage, de la communication et du politique, et porte sur des objets, des époques et des lieux particulièrement variés. Les supports discursifs, de forme écrite ou orale, sont nombreux : discours officiels (discours sur l'état de l'Union, discours des Pères fondateurs de la République), institutionnels (comptes rendus des débats du Parlement), discours

\footnotetext{
Université de Lyon, IEP, UMR 5206 Triangle paul.bacot@sciencespo-lyon.fr EA 3119 CEDITEC

d_desmarchelier@orange.fr Université de Lyon, UMR 5191 ICAR sylviane.remi@univ-lyon2.fr
} 
publics sur des questions de société de dimension nationale ou internationale, documents issus de négociations électorales locales, textes historiques...

Mais l'objectif est toujours le même. Il s'agit de prendre la mesure de la place des nombres dans les discours politiques, et plus spécialement de leur portée argumentative, à travers des études qui nous parlent d'évaluation, de palmarès (Bouchard, 2008), de pourcentages, d'indices, de tableaux et de graphiques, de batailles de chiffres et de luttes des nombres.

\section{Les propriétés du langage des chiffres}

S'il existe bien un langage des chiffres, il faut se demander quelles en sont les propriétés, ce qu'il permet de dire ou de taire, ce que sont ses rapports aux valeurs et aux passions, qui en sont les utilisateurs principaux, quelles stratégies il sert, quelle est la place de la controverse dans le dialogue qu'il génère et, finalement, quel lien particulier l'unit à la chose politique. En somme, il s'agit de dire ce que la politique fait aux chiffres, et ce que les chiffres font à la politique.

Si les chiffres et les nombres sont des concepts de base d'une discipline ayant sa terminologie propre - les mathématiques -, les mots et les symboles qui les expriment font partie du lexique courant et traversent tous les types de discours qui circulent dans la société, du plus familier au plus institutionnel et au plus savant. Dans cette mesure, ils possèdent des valeurs à la fois dénotatives et connotatives.

Les mots chiffre et nombre entrent dans une relation lexicale particulière. La fortune lexicale du mot chiffre ne constitue pas le moindre des paradoxes si l'on rappelle que chiffre (dont la première occurrence en français remonte à 1485) vient du latin médiéval cifra ("zéro »), lui-même emprunté à l'arabe çifr ("vide », «zéro »). Si le mot chiffre, à l'origine, désigne un caractère permettant de former un nombre, il prend par synecdoque, dans les emplois courants, le même sens que nombre. C'est ainsi qu'on peut rencontrer "le chiffre de l'abstention, de la population», "le chiffre d'affaires», «faire du chiffre »... Pour autant, les deux mots ne sont pas toujours des synonymes interchangeables. Nombre, qui, dans son sens courant, exprime la pluralité, tend davantage vers l'expression d'une masse indéterminée et souvent importante. Ainsi l'on peut parler d'un «petit nombre », d'un «grand nombre», d'un «nombre incalculable de victimes» (mais non d'un "chiffre» dans ces contextes) et l'on trouve les expressions « en nombre», «faire nombre», «subir la loi du nombre», etc. Cette distribution permet de distinguer phénomène et entité, ce qu'on retrouve dans l'opposition massif vs comptable. On évoquera «les chiffres du chômage» vs «le nombre de chômeurs» ou encore, «les chiffres de la délinquance» (massif) vs « le nombre d'agressions à caractère raciste» (comptable). 
On distingue traditionnellement les nombres cardinaux et les nombres ordinaux. Les premiers servent à noter des quantités. On les divise en entiers naturels, entiers relatifs, nombres décimaux, nombres rationnels (ou fractionnaires: les deux tiers de, trois-quarts des, etc.). Les seconds traduisent le rang, la position relative, la hiérarchisation : ils se prêtent aux classements, aux palmarès et aux jugements de valeur qui s'y attachent. Ainsi le mot premier prend facilement une valeur positive exprimant l'excellence. Chiffres et nombres peuvent s'écrire sous la forme de mots ou avec des chiffres. Les quantités indéfinies peuvent prendre des formes lexicalisées et non pas chiffrées («une fraction de», «la majorité de », « une quantité de », «un tas de »...). On s’intéressera dans ce numéro à toutes ces formes de quantification et à la dimension spécifiquement linguistique et argumentative qu'elles prennent dans les discours politiques.

Mettre en chiffres, c'est en quelque sorte changer de langage puisque l'on choisit de traduire en signes mathématiques des données d'expérience que l'on pourrait exprimer par des mots. Ce faisant, on passe de la complexité du réel à la complexité du langage mathématique, fondée sur les principes d'une discipline scientifique, mais qui, dans ses résultats, donne de cette réalité une image abstraite, épurée, apparemment simplificatrice, et réductrice. Quelles sont les conditions de production de ce langage? Comment (re)structure-t-il les données qu'il exprime? La quantification «ne fournit pas seulement un reflet du monde (point de vue usuel), mais elle le transforme, en le reconfigurant autrement» (Desrosières, 2008, p.11).

La première question pose le problème de l'adéquation du langage mathématique avec les données qu'on entend lui faire exprimer. Toute donnée, tout référent peut-il être mis en chiffres? Ainsi est-il rare que la quantification s'empare de sentiments, de sensations, d'abstractions tels que l'amour, le plaisir, la douleur, la beauté, même si l'on voit poindre actuellement une revendication de la mesure du bonheur ${ }^{1}$. D'autres données, a priori plus quantifiables, échappent à cette opération tout simplement parce qu'elle n'est pas autorisée ou encore parce qu'il s'agit de pratiques clandestines, comme par exemple le travail au noir ou la fraude fiscale, ou encore parce que le temps a effacé la matière à décompter.

Parmi les données visibles et quantifiables, toutes ne se prêtent pas de manière uniforme à la quantification. Si, comme l'écrit Frege (cité par Saulnier, infra), une fleur a quatre feuilles, elle n'en a pas cinq. La perception des objets concrets, des «entités du premier ordre» (Lyons, 1980, p. 77 et suiv.) effectue une discrétisation des entités qui les prédispose à une catégorisation en langage parlé et à un dénombrement en langage mathématique. Mais il en va différemment des entités du deuxième ordre (toujours au sens de Lyons). Il devient

1. Olivier Zajec, "Mesurer le bonheur?», dans L'Atlas du Monde diplomatique. Mondes émergents, Le Monde diplomatique, hors-série, 2012. 
en effet plus difficile de s'entendre sur l'identification d'événements, de processus, d'états de choses. Si l'on peut compter des humains au même titre que des feuilles, il est plus difficile de s'entendre sur les catégories sociales qu'ils constituent - par exemple, les prostituées (Lilian Mathieu), les consommateurs de drogue (Ivana Obradovic et François Beck) - quand cette catégorisation repose sur l'identification de comportements. Comment définir les types de délit? Une tentative de cambriolage peut-elle être déclassée en simple effraction (Rafaël Cos)? Comment peut-on mettre en chiffres et en proportions le poids politique (métaphorique!) de chaque parti dans le cadre de la négociation d'une liste de coalition aux élections municipales (Nicolas Bué)?

Les statistiques présupposent des agrégats comparables, surtout dans le cadre de vastes études comparatives ainsi que dans les études longitudinales sur de longues périodes. Ainsi, les électeurs du Front national en France et ceux de tel autre parti d'extrême droite dans un autre pays sont-ils interchangeables? Qu'en est-il de ceux du Front national d'aujourd'hui et d'il y a vingtcinq ans? La précocité croissante des consommations de drogue peut-elle être avérée de 1960 à nos jours (I. Obradovic et F. Beck)?

La seconde question qui se pose concerne cette transformation de l'objet mis en chiffres, qui se trouve ainsi discrétisé, catégorisé, dans un processus de naturalisation, de réification, d'objectivation tendant à faire oublier les «conditions d'équivalence qui ont présidé à leur construction» (Supiot, cité par Franck Bessis et Delphine Remillon).

Au-delà de la mise en chiffres, il y a la mise en images, en schémas, en graphiques, en courbes, en tableaux, en camemberts et autres modélisations de toutes sortes. Il s'agit d'une seconde transformation sémiotique, car les chiffres eux-mêmes sont alors iconisés. Ils sont perçus comme de belles images, souvent mises en valeur par une profusion de couleurs. Dans une certaine mesure, on peut dire que cette formalisation constitue un niveau supplémentaire de réduction de la réalité, car ces modélisations ne rendent compte qu'imparfaitement des résultats chiffrés pris en eux-mêmes.

Dans le cadre de cette catégorisation et de cette véritable mutation des données de l'expérience en chiffres, dont on mesure la complexité et la relative fragilité, une question cruciale se pose, qui est celle des sources de cette mise en chiffres. D'où viennent les chiffres, les statistiques, les tableaux, les graphiques, les études transversales et longitudinales qui alimentent tant de discours écrits et oraux de notre communication politique quotidienne? En un mot, quels sont les agents de production des chiffres? Si la naissance des écritures a été accompagnée de comptages divers (des bâtons, des croix, des associations de traits) destinés à quantifier (les réserves de céréales, les productions, le bétail, les morts...), cette production tend, de nos jours, en raison de la sophistication et de la technicisation très grande des outils mathématiques et statistiques, à devenir le domaine exclusif des spécialistes, des savants. Si 
certains de ces « experts», fortement médiatisés, sont connus du grand public à titre individue ${ }^{2}$, les sources des chiffres cités proviennent massivement d'organismes divers : organisations internationales, services ministériels, agences européennes, Conseil d'État, instituts d'études, cabinets d'audit et de conseil, think tanks... Ces structures seront évaluées selon leur degré d'indépendance par rapport à différents types de pouvoir - politique, financier, patronal, syndical... On peut aussi avoir recours, de manière plus informelle, à des rapports d'enquête, des auditions menées en commission, ou des travaux de personnes considérées, à tort ou à raison, comme qualifiées dans le domaine.

Mais la notion de production n'en présente pas moins une certaine ambiguïté. Quand on dit que les experts ou les structures qui encadrent l'expertise produisent les chiffres, cela signifie qu'ils les construisent, qu'ils en sont les concepteurs. Mais ce sont rarement ces concepteurs eux-mêmes qui les introduisent dans l'espace public, c'est-à-dire qui en assurent la diffusion. Ces chiffres sont repris plus ou moins fidèlement, reformatés et propagés à différents niveaux, international ou national, parl'ensemble des responsables, partenaires et acteurs de la vie politique, économique, administrative ou culturelle, à travers une prolifération de textes et discours, écrits et oraux, de genres très différents, empruntant des voies médiatiques très diversifiées. Il convient donc de distinguer, dans la production de chiffres, ce qui est le fait des producteurs-concepteurs et des producteurs-utilisateurs.

La question qui se pose alors est de savoir comment ces deux niveaux d'énonciation s'articulent, de quelle nature sont les références chiffrées qu'introduisent les utilisateurs et quelle en est la légitimité. Dans les canaux de diffusion du mouvement contemporain pour l'abolition de la prostitution, les sources sont quasiment invérifiables, les données étant le plus souvent de seconde main et les références bibliographiques issues de travaux de compilation. De plus, la mise en circulation des chiffres, dont la traçabilité n'est pas toujours assurée, alimente souvent une forte intertextualité qui produit un effet d'unanimité en trompe-l'œil (L. Mathieu). La légitimité de telle ou telle source donne souvent lieu à des controverses, parfois au sein d'une même famille politique. Il en va ainsi des chiffres de la délinquance en France, fournis par les statistiques ministérielles (plus particulièrement par un index statistique, l'État 4001) et par l'Observatoire national de la délinquance et des réponses pénales (ONDRP) : à l'intérieur du PS, Delphine Batho, secrétaire nationale à la sécurité du Parti socialiste de 2003 à 2008 , mettait en avant l'indépendance de l'ONDRP tandis que Jean-Jacques Urvoas, universitaire proche des chercheurs en sciences sociales, qui lui succède en 2009 , dénoncera le caractère trop politique de cet organisme et prônera le recours à l'INSEE (R. Cos).

2. On pense, par exemple, à François Lenglet, invité permanent de l'émission télévisée «Des paroles et des actes» du jeudi soir sur France 2. 


\section{La rhétorique des chiffres}

La disjonction entre concepteurs et consommateurs de chiffres et de statistiques semble être devenue la situation la plus courante, et elle configure de manière spécifique le dispositifénonciatif. En effet, les utilisateurs n'ayant pas la science et les compétences des experts, les discours politiques ne peuvent porter sur les chiffres pris en eux-mêmes, mais doivent se construire à partir et au moyen des chiffres. Auréolés d'un prestige scientifique d'autant plus grand que les acteurs de l'énonciation ne possèdent pas la maîtrise des disciplines dans lesquelles ils s'inscrivent, les chiffres ont alors vocation à se mettre au service de la dimension rhétorique et argumentative des discours politiques. De chiffres-grandeurs, ils accèdent alors au statut de chiffres-valeurs.

Paradoxalement, les chiffres qui sont présumés neutres, exacts et objectifs (Ogien, cité par N. Bué) et se caractérisent donc par la distance et la froideur, vont - en raison même de ces propriétés - se mettre au service des finalités rhétorique, argumentative et polémique des discours politiques.

Les chiffres contribuent d'abord à donner «bonne figure » à ceux qui ont recours à eux. L'ethos prend ici toute sa place. Le prestige accordé aux chiffres donne du locuteur l'image de la rigueur, du sérieux, de la maîtrise de soi et du monde. Les utilisateurs s'approprient en quelque sorte par procuration la puissance de l'objet-chiffre et la science des experts, tout en feignant de s'effacer modestement derrière des formules universalisantes telles que «Tous les experts s'accordent à dire que... », voire derrière les chiffres eux-mêmes, divinement personnifiés comme une sorte de Pythie : "Les chiffres parlent d'euxmêmes et ceux que je cite, parce qu'ils émanent de l'OCDE, ne sont pas contestables. » (Martine Aubry, citée par F. Bessis et D. Remillon)

Mobilisés dans l'expression de dates historiques ponctuant leurs discours, les chiffres ont également contribué à légitimer la parole des orateurs républicains du 19e siècle (1871-1882) (Aude Dontenwille-Gerbaud). Enfin, pris avec une valeur métadiscursive dans des séries numériques qui ont pour but de structurer le discours, leur utilisation «permet d'asseoir l'autorité de l'énonciateur» (Bertrand Richet).

Faire usage de nombres met le locuteur du côté des puissants, de ceux qui savent et qui peuvent. Citant Bourdieu, Hugo Bréant rappelle que l'utilisation des statistiques est à la fois "acte d'autorité » et "acte autorisé».

Si les chiffres, qui font autorité, confèrent assez naturellement cette vertu à ceux qui les utilisent, on pourrait s'étonner de les voir mobilisés au côté des procédés rhétoriques propres au langage parlé. Or, on constate qu'ils sont utilisés dans des discours politiques qui comportent des images, des métaphores, des hyperboles, avec lesquelles ils coexistent et coopèrent harmonieusement à des fins d'expressivité et de persuasion. Plutôt que de viser l'exactitude, les 
chiffres se mettent parfois au service d'une expression hyperbolique qui fait dériver la quantification, à travers la diversité et l'accumulation des mesures, vers le trop, vers «l'infiniment grand» (H. Bréant). Il en est ainsi de la mise en chiffres du droit, qui dénonce «l'inflation normative française» (Rachel Vanneuville) ou des chiffres de la prostitution qui produisent « un effet de vertige » (L. Mathieu). Ils sont alors souvent combinés à des mots exprimant la dramatisation, à des métaphores de maladies ou de catastrophes naturelles. Il arrive même que l'approximation et la fragilité reconnues des chiffres constituent un argument supplémentaire pour affirmer avec force, par exemple, que «la situation [de la prostitution] est certainement plus grave encore que ce que l'on peut en savoir» (idem).

Ces procédés, ainsi que le soulignent plusieurs articles, visent à atteindre l'émotion et à susciter la plus forte indignation, en particulier quand il s'agit de mettre en scène la souffrance humaine ou la mort à des fins d'action militante ou de mobilisation politique comme, par exemple, dans les écrits de Louise Michel sur la Commune de Paris (Sidonie Verhaeghe).

Ainsi, on l'a dit, le chiffre, expression d'une froide rationalité, devient le véhicule privilégié de l'émotion. C'est donc plus le pathos (et l'ethos) que le logos, plus le sentiment que la raison, qui sont les destinataires de l'argument parle nombre.

S'il est vrai que l'on ne peut discuter des chiffres, on peut se demander s'il est possible de discuter les chiffres... Le monde des chiffres se présente comme celui de l'objectivité, mais aussi comme celui de l'universalité : objectivité résultant du protocole gouvernant leur production; universalité garantie par un système de signes translinguistiques et, partant, transculturels. La contestation des nombres mis en avant dans une argumentation ne peut s'opérer qu'avec d'autres nombres, à défaut de quoi le contradicteur donnera l'impression de fuir trop opportunément le terrain de bataille. Mais, on l'a dit, les utilisateurs d'arguments chiffrés ne sont généralement pas eux-mêmes les auteurs des données avancées.

Dès lors, soit l'effet produit par l'usage de l'argument chiffré est tel que sa mise en doute est jugée trop coûteuse par le contradicteur potentiel, soit la contestation ne pourra pas porter sur la valeur intrinsèque des nombres produits, mais sur leur origine. Les débatteurs ayant en quelque sorte externalisé la fabrication de leurs outils argumentatifs en s'en remettant aux experts et à leurs instituts ne seront pas en mesure de « rouvrir les boîtes noires en les historicisant» (Desrosières, 2008, p. 9). Faute de pouvoir reprendre des calculs dont ils n'ont pas la maîtrise - ce que les parlementaires pouvaient encore faire en 1936, en confrontant et en négociant des «calculs de coins de table» (F. Bessis et D. Remillon) -, ils vont devoir contourner l'impuissance mathématique en recourant à la pratique ordinaire de la vigilance sociohistorique. Seront ainsi posées les questions de l'identité et du statut des commanditaires, des 
auteurs et des diffuseurs des données chiffrées devant être contestées - ce qui peut aller jusqu'à l'accusation de manipulation. La légitimité de l'argumentation arithmétique vient-elle du raisonnement qui l'a produite ou de l'autorité de son auteur?

Mais une autre voie s'offre à la remise en cause de l'argumentation par le nombre. Celle qui consiste à invalider le recours aux chiffres pour traiter du problème faisant débat. À la froideur abstraite des statistiques, il pourra être tentant d'opposer la chaleur de l'expérience individuelle concrète : le ressenti contre le calculé (Sophie Saulnier), la personne contre l'agrégat. Même si l'évocation de chiffres bien choisis peut émouvoir, son objectivité revendiquée frappant la subjectivité, elle n'en reste pas moins désincarnée : la statistique présuppose l'effacement de l'humain particulier : "Comment "faire nombre" ? En ne voyant dans chaque objet qu'une unité et rien d'autre. » (Guedj, 2011, p.13)

Pourtant, le poids des chiffres et le choc des nombres sont parfois tels qu'on ne pourra faire mieux que de chercher d'autres statistiques émanant des mêmes sources que les chiffres contestés, pour tenter une mise en contradiction interne : montrer que les données disponibles peuvent conduire aux conclusions les plus opposées. N'entend-on pas quelquefois remettre en cause «les sondages» en s'appuyant sur... un sondage? La mise en contradiction de l'utilisateur d'arguments chiffrés avec lui-même reste une ressource très utile dans les luttes politiques, tout comme le retournement de chiffres pouvant faire l'objet d'appropriations et d'interprétations concurrentes.

Enfin, et peut-être surtout, l'argument chiffré pourra être contesté en tirant profit du point faible du langage statistique tenant, on l'a signalé plus haut, à sa nature même : parler avec des chiffres est toujours réducteur - ce qui peut fonder, sinon une critique radicale, du moins une relativisation de l'argumentation par les nombres. Car chiffrer, c'est cacher : «La numérisation du monde est un appauvrissement.» (Guedj, 2011, p.123) Le discours quantitatif écarte nécessairement tout ce qui n'est pas quantifiable, et tout ce qui ne peut pas être quantifié hic et nunc, quelle que soit la raison de cette impossibilité. Il ramène la diversité à ce qui est commensurable. Il est fondé sur le choix de critères discriminants par nature réducteurs. De plus, le nombre affiché ne dit rien des conditions intellectuelles et sociales de sa production. Il efface la conflictualité dont il résulte pourtant. En somme, on pourra toujours opposer au discours en chiffres qu'argumenter par le nombre, c'est réduire la réalité à ce nombre et prendre ce nombre pour un argument. Mais peut-on argumenter sans réduire?

Il existe bien une analogie entre le travail statistique et le travail politique. Le premier délimite une population et la découpe en catégories en fonction de variables construites à cet effet. Le second délimite une cité et la découpe en camps en fonction d'un clivage produit à cet usage. Le travail statistique comme le travail politique créent ou utilisent des divisions et des regroupements, discriminent pour mieux penser l'unité. Les nombres nous parlent tou- 
jours d'union et de division, et donc d'alliances et d'oppositions. Ils sont tout indiqués pour porter le consensus et le dissensus, la conflictualité et le rassemblement. Prendre la mesure de son camp et du camp adverse, décompter ceux qu'il faut combattre et ceux qu'il faut aider, évaluer ressources et contraintes pour procéder à leur allocation, établir ou contester les hiérarchies : c'est bien ainsi que se construit politiquement le monde, qu'il s'agisse de luttes électorales ou de politiques publiques, de mobilisations collectives ou de relations internationales, de batailles d'idées ou de luttes armées. Mais encore fautil qu'il y ait décompte : d'une certaine façon, dénombrer, c'est reconnaître - reconnaître un groupe et les individus qui le composent, ce qui passera par la dénomination du premier et, partant, des seconds (S. Verhaeghe).

Cette analogie se traduit par l'utilisation du langage des chiffres en politique. Tout démontre l'extraordinaire capacité mobilisatrice des nombres, qu'il s'agisse de dénoncer (la minorité négligeable ou la masse inquiétante) ou de légitimer (l'élite salvatrice ou la majorité imposante). Le nombre semble bien se prêter au figement et constitue donc à ce titre un élément de choix pour la mise en slogan ou en formule. Il contribue à mettre en scène la maîtrise des problèmes, la capacité à les expliquer et à les résoudre. On pourrait dire que la présence de chiffres peut classer un texte « dans la catégorie de ceux qui sont ordinairement exécutoires et exécutés» (Braud, 2002, p. 511). Plus largement, le langage des chiffres permet la prise en main du monde. L'accumulation de chiffres impressionne et rend dérisoire toute forme d'argumentation non quantifiée. La légitimité est du côté de ceux qui dénombrent et qui mesurent, mais aussi du côté de ceux qui font circuler les données chiffrées. Alain Desrosières (2008) nous dit comment «gouverner par les nombres». On peut s'intéresser aussi à ceux qui contestent par le même moyen.

Mais, nous l'avons dit, si le maniement des nombres appelle la révérence, il suscite aussi la méfiance - comme les différentes formes de langage politique, comme la politique et les politiciens : l'ambiguïté semble être une caractéristique majeure du discours politique, qu'il s'écrive avec des chiffres ou avec des lettres.

\section{Références}

BOUCHARD Julie éd., 2008, La communication nombre, Médiation et information (MEI), n०28, décembre.

BRAUd Philippe, 2002, Sociologie politique, Paris, LGDJ (Manuels).

Collectif, 2011, Actes de la recherche en sciences sociales, no 188 (3), Statistiques et sociologie.

DESROSIÈRES Alain, 1993, La politique des grands nombres. Histoire de la raison statistique, Paris, La Découverte. 
Paul Bacot, Dominique Desmarchelier, Sylvianne Rémi-Giraud

- 2008, l'argument statistique, t. I, Pour une sociologie historique de la quantification, t. Il, Gouverner par les nombres, Paris, Presses de l'École des mines.

GuED Denis, 2011 [1996], L'empire des nombres, Paris, La Découverte / Gallimard.

LAscoumes Pierre, Le Galès Patrick éd., 2004, Gouverner par les instruments, Paris, Presses de Sciences Po (Académique).

LYONS John, 1980, Sémantique linguistique, Paris, Larousse. 